Original paper

\title{
Artificial intelligence and the medical physics profession - A Swedish perspective
}

\author{
Jonas Andersson $^{\mathrm{a}, *}$, Tufve Nyholm ${ }^{\mathrm{a}}$, Crister Ceberg ${ }^{\mathrm{b}}$, Anja Almén ${ }^{\mathrm{c}, \mathrm{d}}$, Peter Bernhardt ${ }^{\mathrm{e}, \mathrm{f}}$, \\ Annette Fransson $^{\text {g,h }}$, Lars E. Olsson ${ }^{\mathrm{d}, \mathrm{i}}$

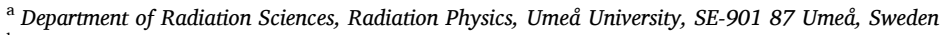 \\ ${ }^{\mathrm{b}}$ Medical Radiation Physics, Department of Clinical Sciences Lund, Lund University, Lund, Sweden \\ ${ }^{c}$ Department of Radiation Protection, Swedish Radiation Safety Authority, Stockholm, Sweden \\ d Department of Translational Medicine, Medical Radiation Physics, Lund University, Malmö, Sweden \\ ${ }^{\mathrm{e}}$ Medical Physics and Biomedical Engineering, Sahlgrenska University Hospital, Gothenburg, Sweden \\ ${ }^{\mathrm{f}}$ Department of Medical Radiation Sciences, Institute of Clinical Science, Sahlgrenska Academy, University of Gothenburg, Gothenburg, Sweden \\ ${ }^{g}$ Department of Medical physics and Nuclear medicine, Karolinska University Hospital, Stockholm, Sweden \\ h Department of Oncology-Pathology, Karolinska Institutet, Stockholm, Sweden \\ ${ }^{i}$ Department of Hematology, Oncology, and Radiation Physics, Skåne University Hospital, Lund, Sweden
}

\section{A R T I C L E I N F O}

\section{Keywords:}

Artificial Intelligence

Medical Physics

Education and Training

\begin{abstract}
A B S T R A C T
Background: There is a continuous and dynamic discussion on artificial intelligence (AI) in present-day society. AI is expected to impact on healthcare processes and could contribute to a more sustainable use of resources allocated to healthcare in the future. The aim for this work was to establish a foundation for a Swedish perspective on the potential effect of AI on the medical physics profession.

Materials and methods: We designed a survey to gauge viewpoints regarding AI in the Swedish medical physics community. Based on the survey results and present-day situation in Sweden, a SWOT analysis was performed on the implications of AI for the medical physics profession.

Results: Out of 411 survey recipients, 163 responded (40\%). The Swedish medical physicists with a professional license believed (90\%) that AI would change the practice of medical physics but did not foresee (81\%) that AI would pose a risk to their practice and career. The respondents were largely positive to the inclusion of AI in educational programmes. According to self-assessment, the respondents' knowledge of and workplace preparedness for AI was generally low.

Conclusions: From the survey and SWOT analysis we conclude that AI will change the medical physics profession and that there are opportunities for the profession associated with the adoption of AI in healthcare. To overcome the weakness of limited AI knowledge, potentially threatening the role of medical physicists, and build upon the strong position in Swedish healthcare, medical physics education and training should include learning objectives on AI.
\end{abstract}

\section{Introduction}

There is a continuous and dynamic discussion on artificial intelligence (AI) in present-day society. It is expected that AI applications will have an impact on healthcare processes and could contribute to a more sustainable use of resources allocated to healthcare in the future [1-3]. There is also an ongoing discussion on the medical physics profession and role of the medical physicist (MP) in the fields of imaging and radiotherapy, which are perceived to be among the first disciplines in healthcare to widely implement AI [4-11]. Existing AI applications have already superseded some analytical or statistical solutions for image reconstruction, segmentation, and registration, as well as clinical decision support (CDS) systems [12]. However, a recent overview of medical AI devices approved by the United States (US) Food and Drug Administration (FDA) has shown that there are limitations in the evaluation process of such devices [13]. Furthermore, there is presently a gap between regulatory aspects and the perspectives of commissioning, quality assurance and control (QA and $\mathrm{QC}$ ) in the clinical implementation of AI

\footnotetext{
* Corresponding author.

E-mail address: jonas.s.andersson@umu.se (J. Andersson).
} 
applications [14-16]. Eventually, this may lead to higher competence requirements on existing healthcare professionals, as well as addition of new competences, e.g., information technology (IT) and AI specialists, and regulatory professionals [14-16]. Another element to the introduction of $\mathrm{AI}$ in healthcare are ethical aspects, e.g., information privacy, which may prove to be the main challenge in avoiding potential harms while maximising the opportunities to cut costs, improving care and patient outcomes, as well as improving the efficiency of entire healthcare systems [17]. In the European Union (EU), regulations relating to radiation safety, medical devices, and information privacy; 2013/59/ Euratom, EU Medical Device Regulation (MDR) and General Data Protection Regulation (GDPR), will govern AI applications that qualify as medical devices within imaging and radiotherapy [18-20]. However, regardless of regulation and medical device technology approval, each healthcare provider is responsible for risk assessment, commissioning, QA, and QC to cover all necessary aspects before new or updated medical devices are implemented in clinical workflows, to ensure quality of care and safety for patients [13-20].

In Europe, the European Federation of Organisations for Medical Physics (EFOMP) started a working group (WG) for Medical Physics and $\mathrm{AI}$ in 2019 with the charge to ensure that the MP professional education, continuous training and competence will follow the global development of AI [21]. Recently, several important reports from this WG and other initiatives, suggesting future pathways for MPs in healthcare have been finalised. This includes a white paper on AI, an international survey on $\mathrm{AI}$ and the MP community, and a proposition on a new curricular and professional programme for AI education and training [22-24]. Further contributions include discussions on consequences from legislation regarding EU MDR and GDPR [15], and how MPs may contribute to procurement, commissioning, and QA of AI applications [16].

Differently from most countries in Europe, Sweden has a five-year undergraduate programme (master's degree) for MPs, which has been revised since the EFOMP survey on education and training in 2008 [25]. The EFOMP survey was a basis for the 2010 policy statement on education and training, which was updated in 2014 [26,27]. The Swedish undergraduate programme is regulated on a national level through learning objectives stated by the Swedish Higher Education Authority [28]. The MP undergraduate programme covers both imaging and radiotherapy physics, including clinical rotations carried out in collaboration with healthcare providers. The curriculum qualifies for a professional medical physics license to practice, which is issued by the Swedish National Board of Health and Welfare [29]. The Swedish Hospital Physicists Association (SSFF) and Swedish Society of Radiation Physics (SFfR) organizes lifelong learning courses on pertinent topics and encourages Swedish MPs to take part in EFOMP's European School for Medical Physics Experts (ESMPE) [30]. ${ }^{1}$ However, presently there are no formal demands on the extent of lifelong learning for MPs in Sweden.

To the best of our knowledge no country in Europe has so far presented a national perspective of $\mathrm{AI}$ and its potential consequences for the medical physics profession. We believe that this perspective can offer valuable insights and contribute to the ongoing discussion. Furthermore, unfortunately Sweden did not participate in the recent EFOMP survey on AI [23]. Instead, we supplemented a survey from the International Organization for Medical Physics (IOMP) to gauge viewpoints regarding AI in the Swedish MP community. The aim for this work was to establish a foundation for a Swedish perspective on the potential effect of AI on the medical physics profession. Therefore, we performed a SWOT analysis (Strengths, Weaknesses, Opportunities and Threats) of the survey results, the present-day situation in Sweden (i.e., MP educational programmes, position in healthcare and radiation safety legislation), and

\footnotetext{
${ }^{1}$ SSFF is an association for organizing MPs, which is a part of a larger union, while SFfR is a scientific organization for engaging scientists in radiation physics and medical physics.
}

the authors' perception of the present evolution of AI in healthcare, academia, and the industry.

\section{Materials and methods}

A group of Swedish MPs, i.e., the authors, was assembled by SFfR with the charge to review and analyse present and emerging applications of AI within the traditional fields of medical physics, the potential overlap between MP tasks and AI applications in healthcare, as well as the viewpoints on AI in the Swedish community. The group consisted of MPs in active clinical duty within both imaging and radiotherapy, working with research and education, with specialization on the regulatory perspective on radiation safety, as well as in managerial positions within healthcare, education, and research.

\subsection{Online survey}

An online survey was sent to recipients affiliated with SSFF. The questions in the IOMP survey were translated to Swedish, in some instances partly reformulated, and supplemented with 12 additional questions identified as important by the authors. The added and reformulated questions were designed to make the survey better adapted to reflect the opinion of the Swedish community. In total there were 29 questions, seven of which were designed to categorize the survey respondents. The survey questions and answer alternatives are listed in Table 1.

In the reporting of results on viewpoints from the respondents, sets of questions $(\mathrm{Q})$ covering a given topic were grouped as:

- AI will change the practise of medical physics (Q1, 2, 14-17, and 20)

- AI should be part of medical physics education and training (Q4, 5, and 6)

- Workplace preparedness for AI (Q21 and 22)

- Basic knowledge of AI (Q7, 8, 11, and 13)

- Advanced knowledge of AI (Q9, 10, 18, and 19)

To further analyse the survey results, the respondents were divided into six different groups according to their replies to Q24-29. In this analysis, only respondents with a professional medical physics license were included (Q23, where multiple answers were possible). The characterizations were:

- Q24. Level of education ( $\mathrm{PhD}$ degree or not)

- Q25. Part- or full-time employment with a university (yes or no)

- Q26. Number of years since receiving professional medical physics license (more vs. less than or equal to 15 years)

- Q27. Main field of practice (imaging or radiotherapy)

- Q28. Gender (male or female)

- Q29. Age (over vs. under or equal to 40 years of age)

To improve the conditions for a statistical analysis of Q1, 2, 4-11 and 13-22, we merged replies "Strongly agree" and "Agree" as Agree and "Strongly disagree" and "Disagree" as Disagree. Statistical significance was investigated with a two-sided Z-test to identify differences between groups of respondents. A significance level of 0.05 without correction for multiple testing was used.

\subsection{SWOT analysis}

A SWOT (Strengths, Weaknesses, Opportunities and Threats) analysis is a common tool for strategic planning [31], traditionally used in the industry but also adopted for organizational development in healthcare and education, e.g., as described by Christiansen (2002), Caruana et al. (2010), and Leiber et al. (2018) [32-34]. The analysis involves identifying strengths and weaknesses in an entity (in this case the medical physics profession) and linking them to perceived 
Table 1

Survey questions and answer alternatives in brackets. Where answer alternatives are not indicated they were [Strongly agree/Agree/Neither agree nor disagree/ Disagree/Strongly disagree]. Questions 1-4, 6-13, 23-25, 28, and 29 were included in the IOMP survey.

1. AI will play an important role in the practice of medical physicists.

2. More and more tasks such as quality control and treatment planning will be performed by AI.

3. In my opinion, AI will threaten/disrupt the medical physicists' practice and career.

4. All medical physicists should acquire at least some basic knowledge of AI.

5. AI should be taught in the undergraduate medical physicist programme.

6. AI should be taught in the postgraduate medical physicist programme (Doctor of Philosophy, $\mathrm{PhD}$ ).

7. I have a basic understanding of AI (relevant to my field).

8. I have working knowledge of AI (relevant to my field).

9. I have relevant skills in AI.

10. I am proficient in AI (able to design, code and implement).

11. I understand the limitations of AI within my field.

12. My skill in AI has been acquired through: [Self-taught/Learned by attending

courses/Learned from postgraduate training/No knowledge].

13. I am ready to learn and apply AI in my practice.

14. AI should not be introduced in healthcare until the black box aspect has become transparent.

15. Within five years, AI solutions will lead to significant reduction of radiation dose in imaging.

16. Medical physicists will work with establishing clinical evidence for new AI applications.

17. Manufacturers of medical devices will take responsibility of all quality control within radiotherapy and nuclear medicine, which is already being implemented for $\mathrm{x}$-ray modalities.

18. I have pertinent knowledge within programming, mathematics, and statistics, which should be sufficient to understand scientific literature on AI (relevant to my field).

19. I have pertinent knowledge to contribute to procurement of medical devices with AI components (e.g., for quality control and image reconstruction).

20. Healthcare providers must hire new staff with AI expertise to solve problems that are presently in the domain of medical physics.

21. Colleagues at my workplace are encouraged to develop their knowledge of AI.

22. At my workplace there is a high level of AI knowledge.

23. My practice can best be described as: [Researcher/Licensed medical physicist/PhD

student/Undergraduate student/Retired/Other] (multiple answers were possible).

24. My level of education is: [PhD degree/Master's degree/Bachelor's degree/Other] (multiple answers were possible).

25. I am employed by: [University/Healthcare/Research institute/Government agency/Regulatory body/Consultancy/Other] (multiple answers were possible).

26 . I have been a licensed medical physicist for (number of years): [0-5 years/6-10 years/11-15 years/16-20 years/Over 21 years/Not licensed].

27. My main practice is within: [Diagnostic x-ray /Nuclear medicine/Radiotherapy/ MRI/Radiation protection/Other].

28. My gender is: [Male/Female/Don't want to specify].

29 . My age is: $[<30$ years/31-40 years/41-50 years/51-60 years/Over 60 years/ Don't want to specify].

opportunities and threats in the surrounding environment [31].

Based on the present-day situation in Sweden (i.e., educational programmes, position in healthcare and radiation safety legislation), the authors' perception on the present evolution of AI in healthcare, academia and the industry, and survey replies from MPs with a professional license, a SWOT analysis was performed on the implications of AI for the medical physics profession.

\section{Results}

\subsection{Online survey}

Out of 411 survey recipients affiliated with SSFF, 163 responded (40\%). Several respondents indicated that they had used the option Neither agree nor disagree when they did not know how to reply to a question, leading to uncertainty in such interpretations below. The respondents' practices were described as: 146 (90\%) licensed MPs, 35 (21\%) researchers, and $14(9 \%) \mathrm{PhD}$ students (Q23, where multiple answers were possible). In the reporting and analysis below, we have only used replies from MPs with a professional license. On their level(s) of education (Q24, where multiple answers were possible), $42 \%$ of the respondents indicated a $\mathrm{PhD}$ degree and $58 \%$ a master's degree. Regarding their employment (Q25, where multiple answers were possible), $88 \%$ of respondents had part- or full-time employment with healthcare and $12 \%$ with universities ( $3 \%$ had neither part-nor full-time employment with healthcare or a university). The respondents' work experience in years after receiving a professional license was reasonably evenly distributed, as shown in Fig. 1 (Q26).

The respondents' main fields of practices (Q27) were radiotherapy (35\%) and diagnostic $\mathrm{x}$-ray (31\%), followed by nuclear medicine (18\%), and MRI (9\%). The gender of respondents was well balanced, $51 \%$ were men and $46 \%$ women, while $3 \%$ opted not to specify their gender (Q28). On the question of their ages (Q29), most respondents answered 31-40 years (35\%), followed by $41-50$ years (30\%), and $51-60$ years $(22 \%)$.

It was clear that the respondents did not think that $\mathrm{AI}$ is a risk to their practice and career, as seen in Fig. 2 (Q3, 81\% Disagree or Strongly disagree).

The survey offered insights on how respondents had acquired their knowledge (if any) of AI (Q12). Almost half of the respondents (44\%) indicated that their present knowledge of AI was self-taught (Fig. 3).

\subsubsection{AI will change the practice of medical physics (Q1, 2, 14-17, and 20)}

A summary of respondents' replies to questions on whether AI will change the practice of medical physics are shown in Fig. 4.

The survey respondents appeared to be convinced that AI will change the practice of medical physics (Q1, 90\% agreed). Traditional core MP tasks, such as QC and treatment planning were perceived to be overtaken by AI solutions by the respondents (Q2, 77\% agreed). On the question whether AI should not be introduced in healthcare until the black box aspect of AI has become transparent (Q14), the respondents were split between positive (30\%) and negative (31\%) replies, while most opted to neither agree nor disagree (39\%). Regarding the MP task of optimization in imaging, the respondents believed that AI will allow for significant reduction of radiation dose in examinations based on ionizing radiation within the next five years (Q15, 40\% agreed), while there were about as many undecided (Q15, 38\% neither agreed nor disagreed) and a minority were negative to this evolution (Q15, 22\% disagreed). More clear indications were given in respondents' viewpoints on that MPs will work with establishing clinical evidence for new AI applications (Q16, 71\% agreed). While most respondents assumed that QC will be overtaken by AI applications, they were divided on whether manufacturers will use such AI applications to take responsibility of QC (Q17, 39\% undecided and 36\% disagree). On whether healthcare providers must hire new staff with AI expertise to work in the present domain of MPs (Q20), the respondents were not in agreement; $38 \%$ believed that this will happen, while $30 \%$ were undecided.

Those most certain that AI will change the practice of medical

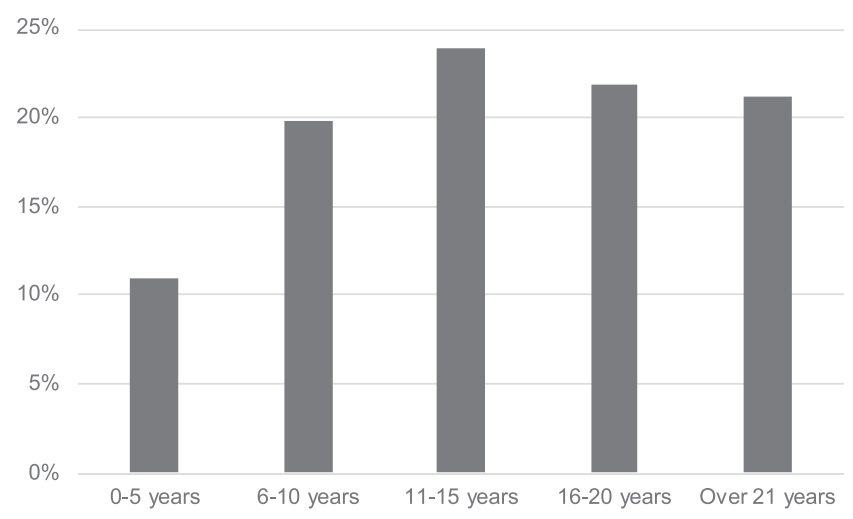

Fig. 1. The result for Q26 "I have been a licensed medical physicist for (number of years):". 


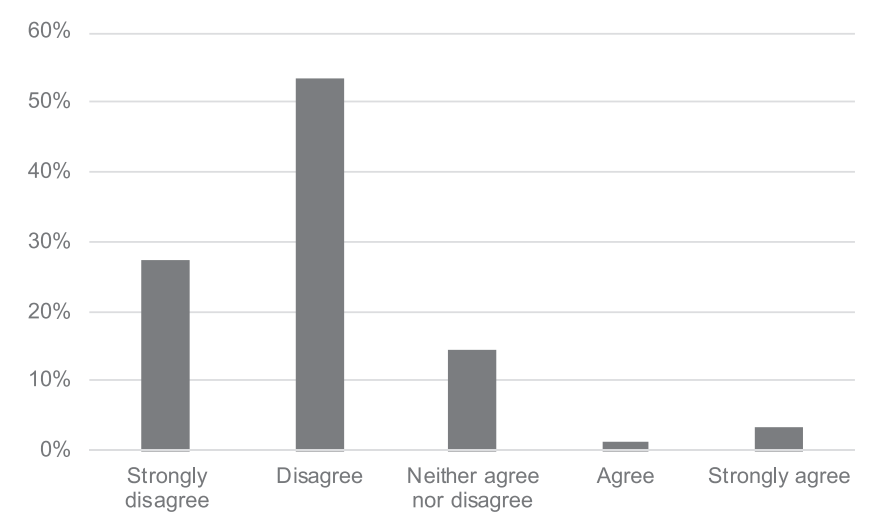

Fig. 2. The result for Q3 "In my opinion, AI will threaten/disrupt the medical physicists' practice and career".

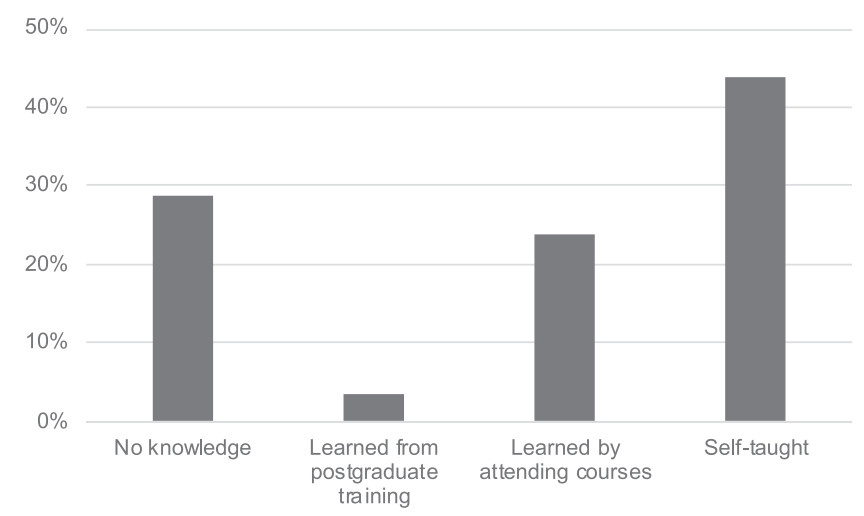

Fig. 3. The result for Q12 "My skill in AI has been acquired through:".

physics (Q1) were respondents with part- or full-time employment at universities ( $\mathrm{p}<0.001)$. MPs with their main field of practice in imaging were most convinced that AI will lead to significant reduction of radiation dose in imaging (Q15, $\mathrm{p}<0.1$; borderline significant [35]). Furthermore, MPs aged 40 years or younger were more frequently of the opinion that healthcare providers must employ staff with AI expertise to solve problems that are presently in the domain of medical physics (Q20, $\mathrm{p}<0.05)$.
3.1.2. AI should be part of medical physics education and training (Q4, 5 , and 6)

A summary of respondents' replies to questions regarding AI education and training for MPs are shown in Fig. 5.

Concerning education and training, a clear majority of respondents were positive to the inclusion of AI in MP educational programmes. They were predominantly of the opinion that all MPs should have basic knowledge about AI (Q4, 87\% agreed), that AI should be a part of undergraduate (Q5, 77\% agreed) and postgraduate (Q6, 68\% agreed) education and training.

For answers to questions on whether AI should be part of medical physics education and training there were no statistically significant differences between different groups of respondents, i.e., all categories of MPs were generally positive to the inclusion of AI in education and training.

\subsubsection{Workplace preparedness for AI (Q21 and 22)}

A summary of respondents' replies to questions on preparedness for AI in their workplaces are shown in Fig. 6.

The respondents were divided, with a preference towards negative, on questions related to their workplace preparedness for AI applications. Regarding whether colleagues at their workplace are encouraged to develop their knowledge of AI (Q21), 37\% agreed while 40\% disagreed.

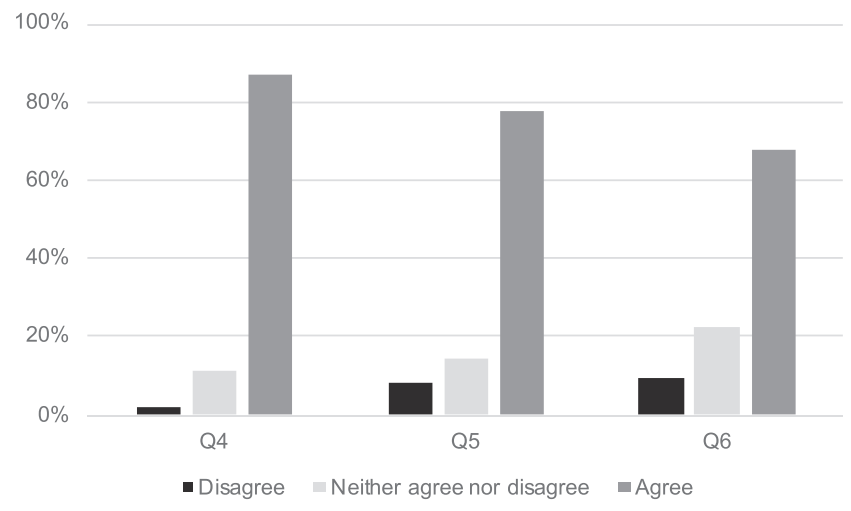

Fig. 5. AI should be part of medical physics education and training (Q4, 5, and 6). The results for Q4 "All medical physicists should acquire at least some basic knowledge of AI", Q5 "AI should be taught in the undergraduate medical physicist programme", and Q6 "AI should be taught in the postgraduate medical physicist programme (Doctor of Philosophy, PhD)".
$100 \%$

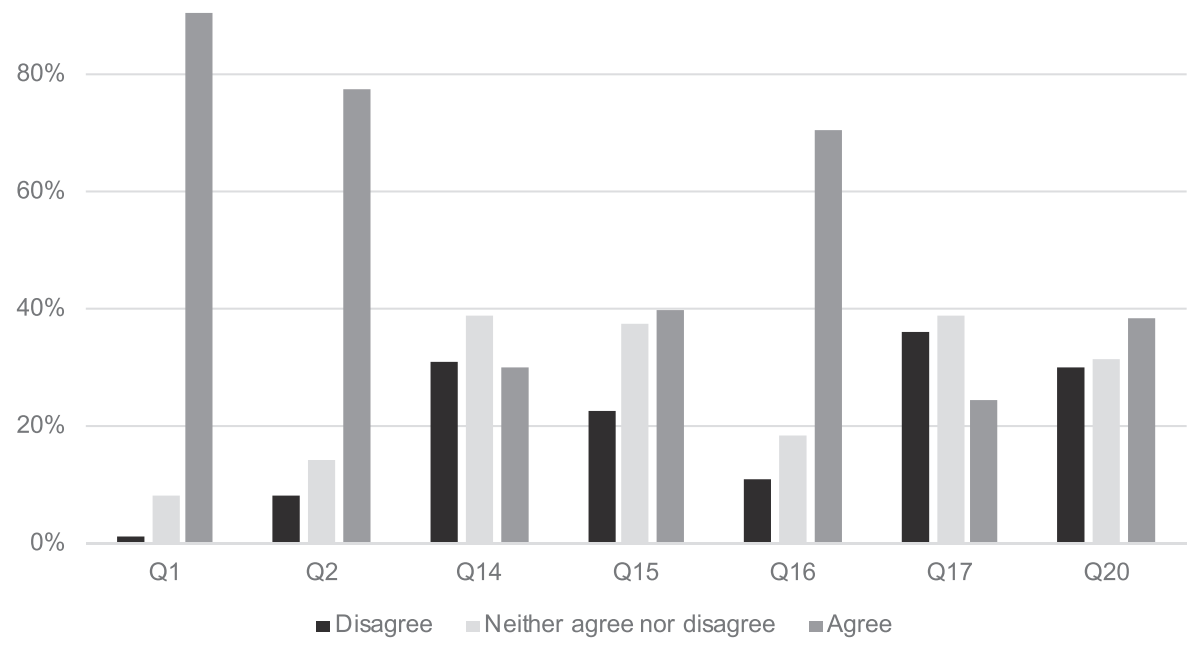

Fig. 4. AI will change the practice of medical physics (Q1, 2, 14-17, and 20). The results for Q1 "AI will play an important role in the practice of medical physicists", Q2 "More and more tasks such as quality control and treatment planning will be performed by AI", Q14 "AI should not be introduced in healthcare until the black box aspect has become transparent", Q15 "Within five years, AI solutions will lead to significant reduction of radiation dose in imaging", Q16 "Medical physicists will work with establishing clinical evidence for new AI applications", Q17 "Manufacturers of medical devices will take responsibility of all quality control within radiotherapy and nuclear medicine, which is already being implemented for $\mathrm{x}$ ray modalities", and Q20 "Healthcare providers must hire new staff with AI expertise to solve problems that are presently in the domain of medical physics". 


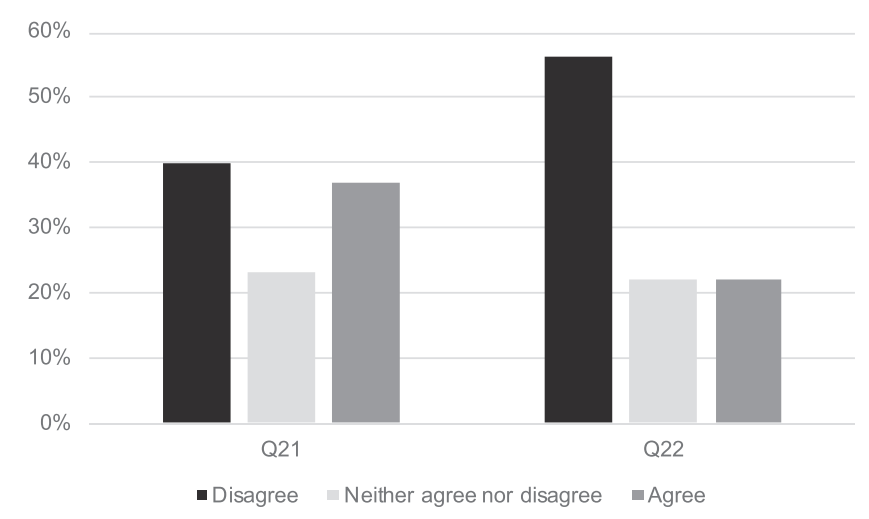

Fig. 6. Workplace preparedness for AI (Q21 and 22). The results for Q21 "Colleagues at my workplace are encouraged to develop their knowledge of AI", and Q22 "At my workplace there is a high level of AI knowledge".

The question on AI competence at respondents' workplaces also yielded responses with an inclination towards negative (Q22), where $56 \%$ indicated that their workplace did not have a high level of AI competence.

On the question if colleagues at their workplaces were encouraged to develop their knowledge of AI (Q21), MPs with part- or full-time employment at universities were distinctly more positive than others $(\mathrm{p}<0.05)$.

\subsubsection{Basic knowledge of $A I(Q 7,8,11$, and 13)}

A summary of respondents' replies to questions on their basic knowledge of AI are shown in Fig. 7.

The respondents' self-assessed basic knowledge of AI varied (Fig. 7). Many were undecided as to their own abilities (24-39\% neither agreed nor disagreed). On whether they had basic knowledge of AI (Q7), 45\% agreed while $31 \%$ disagreed. There was more confidence in that respondents had enough knowledge of AI to work in their field (Q8, 47\% agreed and $18 \%$ disagreed). Respondents were divided on their understanding of the limitations of AI in their respective fields of practice (Q11), where $29 \%$ agreed and $32 \%$ disagreed. The respondents' readiness to learn and apply AI in their practices was also conflicted (Q13), with $37 \%$ positive and $34 \%$ negative.

Men $(\mathrm{p}<0.01)$ and MPs with a PhD degree $(\mathrm{p}<0.01)$ indicated more often than others that they had a basic understanding of AI (Q7). MPs with part- or full-time employment at universities most often indicated that they had a working knowledge of AI (Q8, p $<0.05)$, and that they understood the limitations of AI (Q11, $\mathrm{p}<0.05)$. MPs with a PhD degree ( $\mathrm{p}<0.1$; borderline significant), and even more so, those

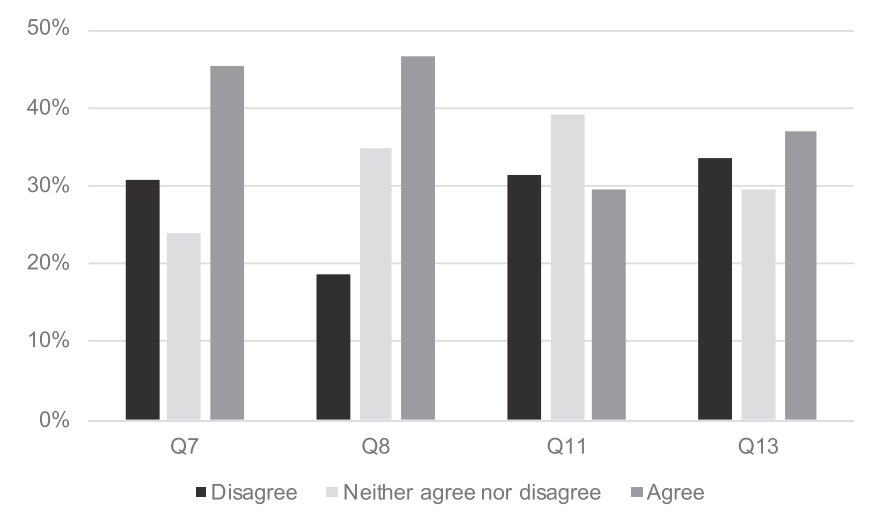

Fig. 7. Basic knowledge of AI (Q7, 8, 11, and 13). The results for Q7 "I have a basic understanding of AI (relevant to my field)", Q8 "I have working knowledge of AI (relevant to my field)", Q11 "I understand the limitations of AI within my field", and Q13 "I am ready to learn and apply AI in my practice". with part- or full-time employment at universities $(\mathrm{p}<0.05)$ indicated that they were ready learn and apply AI in their practices (Q13).

\subsubsection{Advanced knowledge of $A I(Q 9,10,18$, and 19)}

A summary of respondents' replies to questions regarding advanced knowledge of AI are shown in Fig. 8.

On the respondents' self-assessed advanced knowledge of AI, the replies were generally negative (Fig. 8). The exception was their selfassessed knowledge of general programming, mathematics, and statistics, and estimating how such skills could be applied to understand scientific literature on AI (Q18), where $46 \%$ were positive and $33 \%$ negative. Regarding their relevant skills in AI (Q9), $57 \%$ were negative. In their self-assessed knowledge about AI design, code, and implementation (Q10), 90\% were negative, and regarding competence to contribute to tenders on products with AI components (Q19), 51\% were negative.

MPs with part- or full-time employment at universities were most confident that their knowledge within programming, mathematics, and statistics, should be sufficient to understand scientific literature on AI (Q18, p < 0.001), as well as in contributing to procurement of medical devices with AI components (Q19, $\mathrm{p}<0.05)$. Men $(\mathrm{p}<0.1$; borderline significant) and MPs with a PhD degree $(\mathrm{p}<0.1$; borderline significant) also indicated their confidence in these questions, albeit less strongly.

\subsection{SWOT analysis}

The principal strengths, weaknesses, opportunities, and threats identified by the authors in the SWOT analysis on AI and the medical physics profession in Sweden, shown in Table 2, are used to discuss the present work below.

\section{Discussion}

The authors identified two strengths in the SWOT analysis (S1. The $M P$ has a strong professional presence and natural collaboration partners in healthcare, academia, and the industry, and S2. Present radiation safety legislation demands the presence of MPs in healthcare). These strengths were motivated by the long tradition of MPs in Swedish healthcare, where the MP is a link between technology, hardware as well as software, and the clinical or academic use of radiation by other healthcare specialists. This was affirmed by the fact that most survey respondents were convinced of their strong position in healthcare, $81 \%$ disagreed or strongly disagreed with the statement that AI will disrupt or threaten the MPs practice and career. Furthermore, the Swedish implementation of

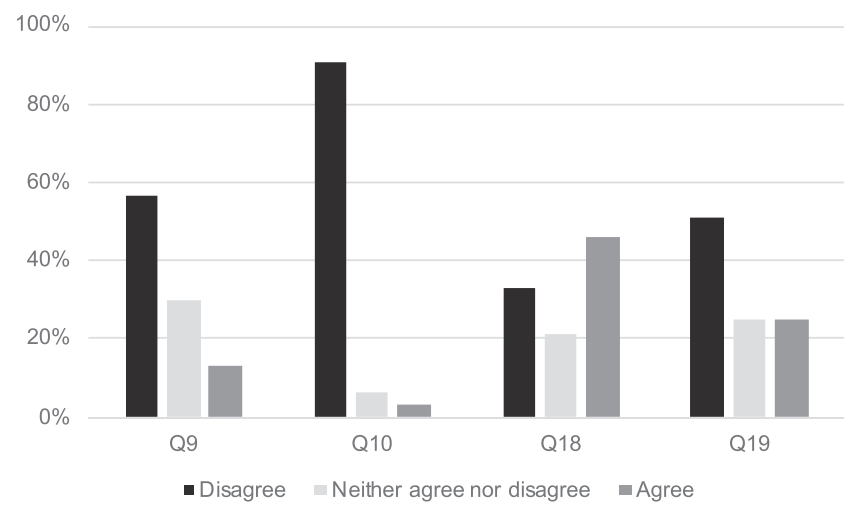

Fig. 8. Advanced knowledge of AI (Q9, 10, 18, and 19). The results for Q9 "I have relevant skills in AI", Q10 "I am proficient in AI (able to design, code and implement)", Q18 "I have pertinent knowledge within programming, mathematics, and statistics, which should be sufficient to understand scientific literature on AI (relevant to my field)", and Q19 "I have pertinent knowledge to contribute to procurement of medical devices with AI components (e.g., for quality control and image reconstruction)". 
Table 2

SWOT analysis results on the implications of AI for the medical physics profession in Sweden.

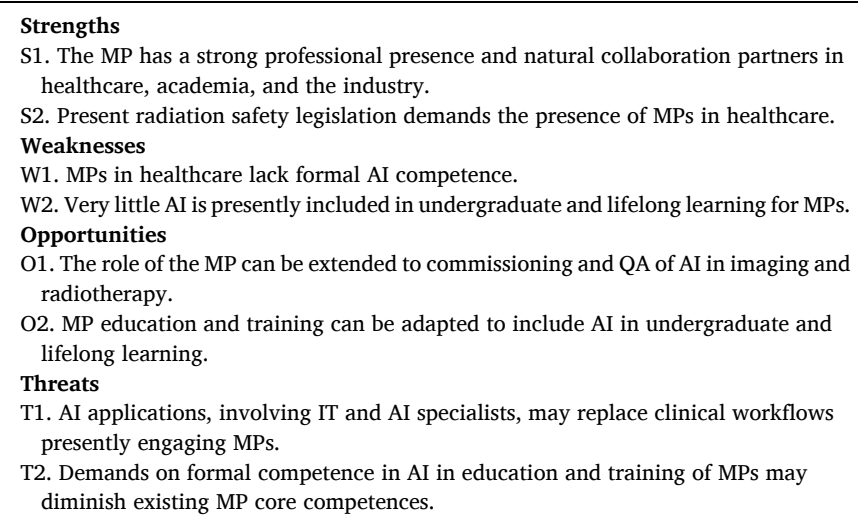

2013/59/Euratom assigns the medical physics expert (MPE), which is in Sweden defined as a licensed MP with at least five years' work experience, a clear role, e.g., in procurement, commissioning, QA, optimization, education and training of other healthcare specialists, and in contributions to healthcare quality management systems (QMS) $[19,36]$. Much of the legislative demands on the practice of MPs in Sweden has to do with medical devices, which increasingly include advanced software components and emerging AI applications. Development of medical devices has historically been driven by rapid technological advances that has had an impact on commissioning, as well as QA and QC. In Sweden, because of this evolution and the digital transformation in healthcare, QC of x-ray equipment is increasingly being carried out by manufacturers or suppliers of medical devices, while healthcare providers continuously review the results for compliance [37]. This change in QA practices was motivated by the facts that while $\mathrm{x}$-ray equipment is becoming increasingly technologically advanced, even hardware technology is now fundamentally digital, and that all manufacturer QC is traceable to standards from the International Electrotechnical Commission (IEC) [38,39]. However, even when not performing the actual QC tasks, MPs in Sweden are required to provide an oversight of the relevance of QC methods used and the compliance to healthcare QMS. The survey respondents were not positive that this change in responsibility for performing QC of equipment would be adopted in radiotherapy and nuclear medicine (only 25\% agreed). Nevertheless, the ongoing digital transformation in healthcare can be expected to further facilitate such QA practices, which may eventually be even more augmented by AI [5].

The identified weaknesses in the SWOT analysis have to do with the lack of AI competence from education in programmes on all levels in Sweden; undergraduate and lifelong learning (W1. MPs in healthcare lack formal AI competence, and W2. Very little AI is presently included in undergraduate and lifelong learning for MPs). The present self-assessed status of basic and advanced knowledge was generally low, but a great majority of survey respondents were of the view that MPs should be expected to have AI competence. It should, however, be noted that MPs with part- or full-time employment at universities, men, and those with $\mathrm{PhD}$ degrees had stronger confidence in their knowledge of AI. There was a minor overrepresentation of $\mathrm{PhD}$ degrees among male (45\%) compared to female (37\%) respondents. Furthermore, the group of respondents with part- or full-time employment at universities consisted of $\mathrm{PhD}$ students with a professional license and MPs with a PhD degree ( $20 \%$ of all respondents with a $\mathrm{PhD}$ degree). Thus, in our analysis there is a small overlap between the group of men with a $\mathrm{PhD}$ degree, and the group of men with part- or full-time employment at a university. The fraction of respondents that self-assessed a high AI competence was very low, and respondents indicating a high general $\mathrm{AI}$ competence in their workplaces were very few. The self-assessed core competence facilitating the ability to build competence within the field of AI through reading scientific literature, as well as the interest in building competence were below $50 \%$. Only about $40 \%$ of respondents indicated that they were encouraged to increase their competence within the field of AI. Furthermore, $44 \%$ of respondents indicated that their present knowledge in AI was self-taught, which can be interpreted as a positive drive among MPs to gain knowledge on AI. However, this may be considered as a risk since such learning can lead to consistent gaps in knowledge. These findings should be contrasted by the overwhelming majority that indicated a belief that AI will be increasingly introduced and adopted to change the practice of medical physics, most strongly so by those with part- or full-time employment at universities. Our interpretation of these results is that it is difficult to anticipate what the actual competence requirements will be, to comprehend the complexity associated with $\mathrm{AI}$, and to predict the actual change and innovation such technology may bring to healthcare. AI competence is probably sometimes seen as the ability to set up, do a hyper-parameter search, and finally to train and test an AI model. However, this ability will probably remain an insignificant part of the work for MPs and may not even guarantee the kind of competence that is needed for the future medical physics profession [40]. In our opinion, pertinent AI competence for MPs would rather include the ability to ask the right questions to vendors about the limitations for an application, and the proficiency to set up routines for commissioning and QA to ensure that such limitations are not breached in clinical practice. Nevertheless, this level of competence is presently not achieved in Swedish MP education and training.

In the SWOT analysis we found that there are good reasons to be optimistic about opportunities for the future of the medical physics profession (O1. The role of the MP can be extended to commissioning and $Q A$ of $A I$ in imaging and radiotherapy, and O2. MP education and training can be adapted to include AI in undergraduate and lifelong learning). Since MPs have a strong professional presence in Sweden and natural collaboration partners in healthcare, academia and the industry, the role could be expanded to encompass AI applications in imaging, radiotherapy, and radiation safety. This is substantiated by the fact that most survey respondents thought that MPs should be involved in establishing clinical evidence for new AI applications (71\%). Furthermore, we agree with Bosmans et al. (2021) in that emerging CDS systems with AI components and virtual clinical trials may be fields where MPs can contribute to development and QA together with physicians (predominantly radiologists), as well as IT and AI specialists, and experts on regulatory matters regarding EU MDR and GDPR [16]. Most survey respondents were very positive to having AI included in both under- and postgraduate MP education and training. In our opinion, there is a good opportunity in the expansion of MP educational programmes to include pertinent knowledge of AI, as previously discussed, which can mitigate the identified weaknesses. This may as well lead to an increased demand for MPs, and further strengthen the role of the MP in healthcare. The authors contacted the four Swedish universities with undergraduate education of MPs and found that there is a high awareness among the programme directors regarding the strong current development in AI, and the consequential imminent need for essential adaptations of the educational programmes. Some teaching and learning activities on AI have been introduced in theoretical and clinical courses, as well as in master's thesis projects and, although the different universities are not yet in the same phase, a national consensus is forming with respect to content. We expect this process to take some time before the actual competence requirements of MPs within AI are realized. Our present position is that MPs are primarily expected to become users of AI, not necessarily software developers, and that the educational focus should be to achieve a knowledge facilitating an understanding of the opportunities and limitations with AI related to the quality of healthcare and patient safety, similarly to what has been suggested by Cui et al. (2020) [40].

Given the ongoing discussion of AI applications, and what is hypothesized about the future, it is reasonable to assume that healthcare 
will go through fundamental changes [1-3]. In the SWOT analysis, the authors found that there may be threats to the Swedish medical physics profession in such future scenarios (T1. AI applications, involving IT and AI specialists, may replace clinical workflows presently engaging MPs, and T2. Demands on formal competence in AI in education and training of MPS may diminish existing MP core competences). It should be noted that MPs aged 40 years or younger most strongly believed that healthcare providers must hire new staff with AI expertise to solve problems that are presently in the domain of medical physics. Beyond CDS systems, other types of decision support systems that may be introduced in the coming years are tools which comprehensively monitor, QC, and optimize entire processes within healthcare $[3,41]$. Potentially eliminating aspects of the traditional roles of healthcare specialists, these tools will be designed to increase both cost efficiency and quality of care [3]. Beyond equipment QC, as previously discussed, examples of such applications within the present field of medical physics may be optimization of imaging protocols, or identification and correction of radiotherapy treatment plans that are likely to fail a QC procedure [5]. Other specialists, with IT and AI competence, are already emerging as future key players in healthcare, as aspects of QA and QC steadily will move towards more data-driven processes $[3,5,41]$. At the same time, another threat to the role of the MP in Sweden is that the introduction of AI in MP undergraduate educational programmes may demand the removal of other core aspects of our present competence profile within physics and mathematics. Nevertheless, it is the programme directors' as well as the authors' opinion that pertinent AI knowledge in MP undergraduate programmes, and more in-depth education and training via lifelong learning, can mitigate the identified threats. A good initiative for improvement of MPs AI competence has already been presented by Zanca et al. (2021) [24], which we think should be adopted and built upon in EFOMP's ESMPE, as digital transformation and AI change healthcare.

A recent international survey performed by EFOMP to assess current perceptions, practices and education needs pertaining to AI in medical physics had 219 respondents from 31 countries [23]. There were similar questions in the Swedish and EFOMP surveys, which makes it possible to compare the respective results. Swedish MPs agree with their European colleagues that AI will change the role of the MP and the medical physics profession, that more AI-specific education and training is required, and that their self-assessed competence with $\mathrm{AI}$ is low. The Swedish and EFOMP results agree in that men have better self-assessed knowledge of AI compared to women. For the Swedish results, a partial explanation to the confidence of men may be the higher fraction of men with $\mathrm{PhD}$ degrees compared to women, as previously discussed. However, it is known that men more often than women overestimate their abilities in self-assessment, especially in an area such as technology, which is commonly perceived as masculine [42-44].

A survey on the impact of AI on radiology has been performed by the European Society of Radiology (ESR) [45]. According to the radiologist respondents in the ESR survey, AI will have a major impact on their profession and mammography reading was identified as the first candidate for applications with CDS systems. About $50 \%$ of the radiologist respondents foresaw increased, while $50 \%$ foresaw decreased, job opportunities due to implementation of AI applications. Apparently, there is uncertainty among radiologists regarding their professional future in different scenarios of AI implementation. The impact of AI on the profession is regarded similarly high by the Swedish MPs according to our survey. However, our respondents had less concerns of the future changes on job opportunities and considered AI more as an opportunity than a threat. All respondents (100\%) in the ESR survey thought that they should be involved in validation and development of AI applications, why training programmes should be adopted to further educate trainees and clinical radiologists on AI [45]. The radiologists' viewpoints on education and training, and on working with establishing clinical evidence for AI applications agree with the Swedish MP survey respondents. In the opinion of the authors, this is a very positive indication, since the natural collaboration between MPs and radiologists can be further built upon with increasing adoption of $\mathrm{AI}$ in healthcare.

\section{Conclusions}

From viewpoints in survey responses from Swedish MPs and the SWOT analysis we conclude that AI will change the medical physics profession and that there are good opportunities for the profession associated with the adoption of AI applications in healthcare. In general, the survey respondents indicated that their workplaces are not well prepared for the perceived AI revolution and that their knowledge of AI, both basic and advanced, was self-assessed to be low. To overcome the weakness of limited knowledge, potentially threatening the professional role of the MP, and build upon the long tradition and present-day strong position of Swedish MPs in healthcare, learning objectives on knowledge of AI should become part of medical physics undergraduate education and training as well as lifelong learning. These conclusions share common grounds with those of other MPs in Europe, as well as European radiologists' viewpoints on their profession and AI.

\section{Funding}

The authors disclose receipt of financial support for publication of this article: This work was supported by the Swedish Society of Radiation Physics.

\section{Declaration of Competing Interest}

The authors declare that they have no known competing financial interests or personal relationships that could have appeared to influence the work reported in this paper.

\section{Acknowledgements}

The authors would like to thank the Swedish Society of Radiation Physics for their support and the Swedish Hospital Physicists Association for allowing us to use their e-mail list to disseminate the survey used in the present work. The authors are also grateful to the International Organization for Medical Physics for sharing their original survey questions on the relevance and impact of AI on the medical physics profession.

\section{References}

[1] Miller DD, Brown EW. Artificial intelligence in medical practice: the question to the answer? Am J Med 2018;131(2):129-33. https://doi.org/10.1016/j. amjmed.2017.10.035.

[2] Noorbakhsh-Sabet N, Zand R, Zhang Y, Abedi V. Artificial intelligence transforms the future of health care. Am J Med 2019;132(7):795-801. https://doi.org/ 10.1016/j.amjmed.2019.01.017.

[3] Shilo S, Rossman H, Segal E. Axes of a revolution: challenges and promises of big data in healthcare. Nat Med 2020;26(1):29-38. https://doi.org/10.1038/s41591019-0727-5.

[4] El Naqa I, Das S. The role of machine and deep learning in modern medical physics. Med Phys 2020;47(5):e125-6. https://doi.org/10.1002/mp.14088.

[5] Tang X, Wang B, Rong Yi. Artificial intelligence will reduce the need for clinical medical physicists. J Appl Clin Med Phys 2018;19(1):6-9. https://doi.org/ 10.1002/acm2.12244.

[6] Nensa F, Demircioglu A, Rischpler C. Artificial intelligence in nuclear medicine. J Nucl Med 2019;60(2):29S-37S. https://doi.org/10.2967/jnumed.118.220590.

[7] Jha S, Cook T. Artificial intelligence in radiology - the state of the future. Acad Radiol 2020;27(1):1-2. https://doi.org/10.1016/j.acra.2019.11.003.

[8] Thompson RF, Valdes G, Fuller CD, Carpenter CM, Morin O, Aneja S, et al. Artificial intelligence in radiation oncology: a specialty-wide disruptive transformation? Radiother Oncol 2018;129(3):421-6. https://doi.org/10.1016/j. radonc.2018.05.030.

[9] Sahiner B, Pezeshk A, Hadjiiski LM, Wang X, Drukker K, Cha KH, et al. Deep learning in medical imaging and radiation therapy. Med Phys 2019;46(1):e1-36. https://doi.org/10.1002/mp.13264.

[10] Lee J-G, Jun S, Cho Y-W, Lee H, Kim GB, Seo JB, et al. Deep learning in medical imaging: general overview. Korean J Radiol 2017;18(4):570. https://doi.org/ 10.3348/kjr.2017.18.4.570. 
[11] Lundervold AS, Lundervold A. An overview of deep learning in medical imaging focusing on MRI. Z Med Phys 2019;29(2):102-27. https://doi.org/10.1016/j. zemedi.2018.11.002.

[12] The U.S. Food and Drug Administration (FDA) cleared AI algorithms. The American College of Radiology (ACR). https://models.acrdsi.org. Accessed 21 May 2021.

[13] Wu E, Wu K, Daneshjou R, Ouyang D, Ho DE, Zou J. How medical AI devices are evaluated: limitations and recommendations from an analysis of FDA approvals. Nat Med 2021;27(4):582-4. https://doi.org/10.1038/s41591-021-01312-x.

[14] Pesapane F, Volonté C, Codari M, Sardanelli F. Artificial intelligence as a medical device in radiology: ethical and regulatory issues in Europe and the United States. Insights Imaging 2018;9(5):745-53. https://doi.org/10.1007/s13244-018-0645-y.

[15] Beckers R, Kwade Z, Zanca F. The EU medical device regulation: implications for artificial intelligence-based medical device software in medical physics. Phys Med 2020;83:1-8. https://doi.org/10.1016/j.ejmp.2021.02.011.

[16] Bosmans H, Zanca F, Gelaude F. Procurement, commissioning, and QA of AI based solutions: an MPE's perspective on introducing AI in clinical practice. Phys Med 2021;83:257-63. https://doi.org/10.1016/j.ejmp.2021.04.006.

[17] Morley J, Machado CCV, Burr C, Cowls J, Joshi I, Taddeo M, et al. The ethics of AI in health care: a mapping review. Soc Sci Med 2020;260:113172. https://doi.org/ 10.1016/j.socscimed.2020.113172.

[18] Council of the European Union. Regulation (EU) 2017/745 of the European Parliament and of the Council of 5 April 2017 on medical devices, amending Directive 2001/83/EC, Regulation (EC) No 178/2002 and Regulation (EC) No 1223/2009 and repealing Council Directives 90/385/EEC and 93/42/EEC. http s://eur-lex.europa.eu/eli/reg/2017/745/2017-05-05. Accessed 21 May 2021.

[19] Council of the European Union. Council Directive 2013/59/Euratom of 5 December 2013 laying down basic safety standards for protection against the dangers arising from exposure to ionising radiation, and repealing Directives 89/ 618/Euratom, 90/641/Euratom, 96/29/Euratom, 97/43/Euratom and 2003/122 Euratom. https://eur-lex.europa.eu/eli/dir/2013/59/oj. Accessed 21 May 2021.

[20] Council of the European Union. Regulation (EU) 2016/679 of the European Parliament and of the Council of 27 April 2016 on the protection of natural persons with regard to the processing of personal data and on the free movement of such data, and repealing Directive 95/46/EC (General Data Protection Regulation). https://eur-lex.europa.eu/eli/reg/2016/679/oj. Accessed 21 May 2021.

[21] The European Federation of Organisations for Medical Physics (EFOMP). Current Working Groups. https://www.efomp.org/index.php?r=pages\&id=worki ng-groups. Accessed 21 May 2021.

[22] Kortesniemi M, Tsapaki V, Trianni A, Russo P, Maas A, Källman H-E, et al. The European Federation of Organisations for Medical Physics (EFOMP) White Paper: big data and deep learning in medical imaging and in relation to medical physics profession. Phys Med 2018;56:90-3. https://doi.org/10.1016/j.ejmp.2018.11.005.

[23] Diaz O, Guidi G, Ivashchenko O, Colgan N, Zanca F. Artificial intelligence in the medical physics community: an international survey. Phys Med 2021;81:141-6. https://doi.org/10.1016/j.ejmp.2020.11.037.

[24] Zanca F, Hernandez-Giron I, Avanzo M, Guidi G, Crijns W, Diaz O, et al. Expanding the medical physicist curricular and professional programme to include Artificial Intelligence. Phys Med 2021;83:174-83. https://doi.org/10.1016/j. ejmp.2021.01.069.

[25] Eudaldo T, Olsen K. The present status of Medical Physics Education and Training in Europe: an EFOMP survey. Phys Med 2008;24(1):3-20. https://doi.org/ 10.1016/j.ejmp.2007.09.022.

[26] Eudaldo T, Olsen K. The European Federation of Organisations for Medical Physics. Policy Statement No. 12: the present status of Medical Physics Education and
Training in Europe. New perspectives and EFOMP recommendations. Phys Med 2010;26(1):1-5. https://doi.org/10.1016/j.ejmp.2009.02.005.

[27] Caruana CJ, Christofides S, Hartmann GH. European Federation of Organisations for Medical Physics (EFOMP) Policy Statement 12.1: Recommendations on Medical Physics Education and Training in Europe 2014. Phys Med 2014;30(6):598-603. https://doi.org/10.1016/j.ejmp.2014.06.001.

[28] The Swedish Higher Education Authority (UKÄ). https://english.uka.se/. Accessed 21 May 2021.

[29] The Swedish National Board of Health and Welfare. https://www.socialstyrelsen. se/en/. Accessed 21 May 2021.

[30] The European Federation of Organisations for Medical Physics (EFOMP). European School for Medical Physics Experts (ESMPE). https://www.efomp.org/index.php? $\mathrm{r}=$ pages\&id=esmpe-about. Accessed 21 May 2021.

[31] Piercy N, Giles W. Making SWOT analysis work. Mark Intell and Plan 1989;7(5/6): 5-7. https://doi.org/10.1108/EUM0000000001042.

[32] Christiansen T. A SWOT analysis of the organization and financing of the Danish health care system. Health Policy 2002;59(2):99-106. https://doi.org/10.1016/ S0168-8510(01)00200-7.

[33] Caruana CJ, Wasilewska-Radwanska M, Aurengo A, Dendy PP, Karenauskaite V, Malisan MR, et al. A comprehensive SWOT audit of the role of the biomedical physicist in the education of healthcare professionals in Europe. Phys Med 2010;26 (2):98-110. https://doi.org/10.1016/j.ejmp.2009.08.001.

[34] Leiber T, Stensaker B, Harvey LC. Bridging theory and practice of impact evaluation of quality management in higher education institutions: a SWOT analysis. Eur J High Educ 2018;8(3):351-65. https://doi.org/10.1080/ 21568235.2018.1474782.

[35] Hackshaw A, Kirkwood A. Interpreting and reporting clinical trials with results of borderline significance. BMJ 2011;343:d3340. https://doi.org/10.1136/bmj. d3340.

[36] The Swedish Radiation Safety Authority. Regulations on Medical Exposures (In Swedish). https://www.stralsakerhetsmyndigheten.se/publikationer/foreskrifter /ssmfs-2018/ssmfs-20185/. Accessed 21 May 2021.

[37] Andersson J, Granberg C, Riklund K. A novel system for quality assurance of radiology equipment. EuroSafe Imaging 2018/ESI-0064. doi: 10.1594/esi2018/ ESI-0064.

[38] The International Electrotechnical Commission (IEC). https://www.iec.ch/h omepage. Accessed 21 May 2021.

[39] Sundström H, Sjöberg J, Andersson J. A Swedish implementation of manufacturer specific quality assurance for radiological equipment. EuroSafe Imaging 2020/ESI11538. doi: 10.26044/esi2020/ESI-11538.

[40] Cui S, Tseng HH, Pakela J, Ten Haken RK, El Naqa I. Introduction to machine and deep learning for medical physicists. Med Phys 2020;47(5):e127-47. https://doi. org/10.1002/mp.14140.

[41] Benke K, Benke G. Artificial intelligence and big data in public health. Int J Environ Res Public Health 2018;15(12):2796. https://doi.org/10.3390/ijerph15122796.

[42] Beyer S, Bowden EM. Gender differences in self-perceptions: convergent evidence from three measures of accuracy and bias. Pers Soc Psychol Bull 1997;23(2): 157-72. https://doi.org/10.1177/0146167297232005.

[43] Moore DA, Healy PJ. The trouble with overconfidence. Psychol Rev 2008;115(2): 502-17. https://doi.org/10.1037/0033-295X.115.2.502.

[44] Amirkhanyan H, Krawczyk MW, Wilamowski M, Schwieren C. Gender inequality and national gender gaps in overconfidence. PLOS ONE 2021;16(4):e0249459. https://doi.org/10.1371/journal.pone.0249459.

[45] The European Society of Radiology (ESR). Impact of artificial intelligence on radiology: a EuroAIM survey among members of the European Society of Radiology. Insights Imaging 2019;10(1):105. doi: 10.1186/s13244-019-0798-3. 